Review Article

\title{
Treatment with Antiangiogenic Drugs in Multiple Lines in Patients with Metastatic Colorectal Cancer: Meta-Analysis of Randomized Trials
}

\author{
R.-D. Hofheinz, ${ }^{1}$ U. Ronellenfitsch, ${ }^{2}$ S. Kubicka, ${ }^{3}$ A. Falcone, ${ }^{4}$ \\ I. Burkholder, ${ }^{5}$ and U. T. Hacker ${ }^{6}$ \\ ${ }^{1}$ Interdisziplinäres Tumorzentrum, Universitätsmedizin Mannheim, University of Heidelberg, Mannheim, Germany \\ ${ }^{2}$ Department of Surgery, Universitätsmedizin Mannheim, University of Heidelberg, Mannheim, Germany \\ ${ }^{3}$ Krebszentrum Reutlingen, Reutlingen, Germany \\ ${ }^{4}$ University of Pisa, Pisa, Italy \\ ${ }^{5}$ Department of Nursing and Health, University of Applied Sciences of the Saarland, Saarbrücken, Germany \\ ${ }^{6}$ University Cancer Center Leipzig (UCCL), Universitätsklinikum Leipzig, Leipzig, Germany
}

Correspondence should be addressed to R.-D. Hofheinz; ralf.hofheinz@umm.de

Received 14 March 2016; Accepted 19 July 2016

Academic Editor: Alessandro Passardi

Copyright (c) 2016 R.-D. Hofheinz et al. This is an open access article distributed under the Creative Commons Attribution License, which permits unrestricted use, distribution, and reproduction in any medium, provided the original work is properly cited.

\begin{abstract}
Background. In metastatic colorectal cancer (mCRC), continuing antiangiogenic drugs beyond progression might provide clinical benefit. We synthesized the available evidence in a meta-analysis. Patients and Methods. We conducted a meta-analysis of studies investigating the use of antiangiogenic drugs beyond progression. Eligible studies were randomized phase II/III trials. Primary endpoints were overall survival (OS) and progression-free survival (PFS). Secondary endpoints were the impact of continuing antiangiogenic drugs (i) in subgroups, (ii) in different types of compounds targeting the VEGF-axis (monoclonal antibodies versus tyrosine kinase inhibitors), and (iii) on remission rates and prevention of progression. Results. Eight studies (3,668 patients) were included. Continuing antiangiogenic treatment beyond progression significantly improved PFS (HR 0.64; 95\%-CI, 0.55-0.75) and OS (HR 0.83; 95\%-CI, 0.76-0.89). PFS was significantly improved in all subgroups with comparable HR. OS was improved in all subgroups stratified by age, gender, and ECOG status. The rate of patients achieving at least stable disease was improved with an OR of 2.25 (95\%-CI, 1.41-3.58). Conclusions. This analysis shows a significant PFS and OS benefit as well as a benefit regarding disease stabilization when using antiangiogenic drugs beyond progression in mCRC. Future studies should focus on the optimal sequence of administering antiangiogenic drugs.
\end{abstract}

\section{Introduction}

First-line chemotherapy for metastatic colorectal cancer $(\mathrm{mCRC})$ is frequently combined with antiangiogenic agents, namely, bevacizumab, a monoclonal antibody targeting vascular endothelial growth factor (VEGF) A. This is based on the results of several studies demonstrating a benefit in progression-free survival [1-4]. However, a statistically significant prolongation of overall survival has only been observed in one of these first-line studies [2] raising the question how long the optimum duration of the use of antiangiogenic drugs should be.
Prolonged duration of bevacizumab treatment until definitive progression has been shown to improve progressionfree survival in the phase 3 NO16966 trial which investigated the addition of bevacizumab to oxaliplatin-based first-line regimens [3]. Moreover, two observational studies showed a correlation between the use of bevacizumab beyond progression and improved overall survival in advanced colorectal cancer $[5,6]$. Preclinical data strongly suggest that continued antiangiogenic treatment beyond progression might provide antitumor efficacy even in further lines of treatment $[7,8]$.

These observations led to the setup of clinical trials, which investigated the use of bevacizumab beyond progression in 
patients who had undergone a bevacizumab-based first-line treatment. Two trials (TML [9] and BEBYP [10,11]), although using different inclusion criteria and endpoints, unequivocally demonstrated that the continued use of bevacizumab beyond progression improved PFS (TML and BEBYP) and overall survival (TML).

More recently, randomized trials investigated other drugs with antiangiogenic properties in second and further lines of treatment in patients with metastatic colorectal cancer after pretreatment with bevacizumab-based regimens. For instance, the phase-3 VELOUR trial investigated the addition of aflibercept (a fusion protein trapping VEGF-A, VEGF$\mathrm{B}$, and placental growth factor $[\mathrm{PlGF}]$ ) in combination with 5-fluorouracil and irinotecan (FOLFIRI) treatment in patients who had been pretreated with oxaliplatin-based regimens [12]. It was demonstrated that the addition of aflibercept improved progression-free and overall survival. Notably, this also held true for the patients with bevacizumabpretreatment [13]. The concept of continued use of antiangiogenic drugs has also been demonstrated in the CORRECT study and the CONCUR study in which treatment with regorafenib-a multikinase inhibitor targeting among others VEGF-receptor 2-was superior to best supportive care in patients who had been pretreated with all active drugs including bevacizumab $[14,15]$. Taken together, data from recent studies suggest that prolonged duration of antiangiogenic treatment might be associated with improved outcome in patients with metastatic colorectal cancer.

In the present meta-analysis we sought to investigate the concept of treatment with antiangiogenic drugs in multiple lines beyond progression by analyzing aggregate data of randomized trials. Special emphasis was given on describing potential improvements of progression-free and overall survival related to specific subgroups including KRAS wildtype patients.

\section{Patients and Methods}

2.1. Objectives of Meta-Analysis and Eligibility Criteria. Primary objective of the present analysis was to investigate progression-free survival (PFS) and overall survival (OS) in patients with metastatic colorectal cancer who had been pretreated with an antiangiogenic treatment and underwent antiangiogenic treatment beyond progression.

Secondary objectives were to assess the effects of the continued or repeated antiangiogenic treatment in subgroups (stratified by age, sex, ECOG status, and tumor KRAS mutational status) and in studies using anti-VEGF treatment (i.e., bevacizumab and aflibercept) versus tyrosine kinase inhibitors (TKI). Moreover, we investigated the response rates (i.e., the rate of evaluable patients achieving complete or partial remissions) and the rate of "tumor stabilization," that is, the rate of evaluable patients without primary progression while receiving treatment.

Only randomized phase II and III trials were included in the current meta-analysis. The inclusion of subgroups of randomized trials was allowed provided sufficient information was given in the trial reports. Only studies performed with the approval of an appropriate ethics committee and conducted in compliance with the declaration of Helsinki were included in this meta-analysis.

Antiangiogenic treatment was defined as the use of drugs targeting at least one important angiogenic pathway, for instance, monoclonal antibodies targeting VEGF or VEGFreceptors, or (multi)TKI targeting angiogenic pathways.

2.2. Information Sources, Search Strategy, and Study Selection. Searches in PubMed and proceedings of international meetings were conducted to identify studies with information relevant for the current analysis.

Eligible studies were phase II or III, randomized, controlled trials comparing (i) antiangiogenic drugs in combination with either active treatment (i.e., chemotherapy) or placebo with (ii) active treatment or placebo alone in patients who had previously been treated with antiangiogenic drugs for metastatic colorectal cancer. We used MeSH and full-text search terms for metastatic colorectal cancer and molecular targeted therapies, limiting our results to English language articles published in PubMed between January 1, 2007, and October 11, 2015. For PubMed, the search was (((("molecular targeted therapy" [All Fields] OR ("molecular" [All Fields] AND "targeted" [All Fields]) AND ("therapy" [All Fields] OR "therapies" [All Fields]) AND ("colorectal neoplasms" [All Fields] OR "colorectal cancer" [All Fields]) OR ("colorectal" [All Fields] AND "cancer" [All Fields]) AND ("randomized" [All Fields] OR "randomized study" [All Fields]) AND English [lang])))).

In addition to computerized search, references of retrieved papers were also screened for missing trials.

To minimize publication bias we conducted a manual search of conference abstracts. For conferences, the search was "colorectal cancer" or "advanced colorectal cancer", manually limited to abstracts on targeted therapies. The proceedings of the following meetings were examined for presented abstracts limiting the search to the years 20072016: (i) American Society of Clinical Oncology (ASCO) annual meetings; (ii) ASCO Gastrointestinal Cancer Symposium; (iii) European Society for Medical Oncology (EMSO) and European multidisciplinary cancer congress (ECCO) meetings; (iv) World Congress on Gastrointestinal Cancer. Two independent reviewers (RDH, UR, or UH) assessed title, keywords, and abstracts of retrieved studies. If studies met the inclusion criteria, they assessed full texts and mutually decided on inclusion.

2.3. Data Collection. Data extraction was conducted independently by three investigators (RDH, UR, and $\mathrm{UH}$ ) in accordance with the Preferred Reporting Items for Systematic Reviews and Meta-Analyses (PRISMA) guidance [19]. For each study the following information was extracted: publication or presentation date, first author's last name, sample size/sample size of subgroup of interest, primary endpoints, information pertaining to study design, PFS, and OS as well as response definition, regimens used, line of treatment, number of outcome events, data on PFS, OS, response to treatment, and data of subgroups of interest. 
TABLE 1: Overview of studies included in meta-analysis.

\begin{tabular}{lcccc}
\hline Study & First author/year & $\begin{array}{c}\text { Investigational } \\
\text { treatment }\end{array}$ & Control treatment & $\begin{array}{c}\text { Primary endpoint } \\
\text { patients (ITT) } \\
\text { TML }\end{array}$ \\
\hline active/control)
\end{tabular}

2.4. Synthesis of Results, Statistical Methods, and Analyses. The impact of antiangiogenic treatment on overall survival (OS) and progression-free survival (PFS) was measured in terms of the hazard ratio (HR). In all studies included in this meta-analysis, the HR was calculated as ratio of the active therapy divided by the hazard of the control group. Therefore, a HR lower than 1 indicates a benefit of the active treatment, whereas a HR higher than 1 indicates a higher risk of death or progression of the active treatment, respectively. The estimates of the HR were extracted directly from the publications and variance of the estimates was calculated from published confidence intervals of the HR. For the TML study, unstratified as well as stratified HRs were reported. As the unstratified analysis was the primary analysis of this study, the unstratified estimates were included in the meta-analysis.

Heterogeneity of the individual HRs was tested using Cochran's Q statistic [19]. If heterogeneity was not detected at the $10 \%$ significance level, the fixed effects model was used. If the test for heterogeneity was significant, the overall HR was calculated using the random effects model [20]. Heterogeneity was quantified by $I^{2}$ coefficient measuring the percentage of total variation across studies that is due to heterogeneity rather than by chance.

Meta-analysis of the remission, progression, and rates of "tumor stabilization" was performed using the same statistical methods described above. In the remission analysis and in the progression analysis patients with unknown status were counted as progressive. Finally, in the "prevention of progression" (POP) analysis patients with unknown status were excluded.

ML-estimates of the odds ratio (OR) were determined from reported contingency tables and the within-trial variance was computed from the inverse of the matrix of second derivatives of the log-likelihood (Woolf's formula).

\section{Results}

3.1. Selected Trials. The Medline search was done on June 15, 2016. It resulted in 3,252 articles. Hand-searches of conference proceedings were conducted including all conferences until June 2016.

Based on the criteria defined above the following eight studies were selected for inclusion into the current metaanalysis (PRISMA diagram; cf. Figure 1): TML trial [9], BEBYP trial [10,11], subgroup of VELOUR study [12], CORRECT trial [14], subgroup of AGITG CO.20 trial [16], a subgroup of patients treated within a randomized phase II trial (FOSCO) investigating the addition of sorafenib to second-line chemotherapy [17], subgroups of the CONCUR study [15], and the RAISE study [18].

Trial characteristics are depicted in Table 1 . All trials were evaluable for PFS and OS.

Table 2 shows the number of patients evaluable from respective trials for the determination of primary and secondary outcome parameters.

3.2. Combined Analysis: Primary Outcomes-ProgressionFree and Overall Survival. The use of antiangiogenic drugs beyond progression improved progression-free survival and overall survival over control (i.e., best supportive care or active treatment alone). The hazard ratio for overall survival $(n=3,668)$ was 0.83 (95\%-CI, 0.76-0.89); (Figure 2$)$. The test for heterogeneity was significant $\left(p=0.010 ; I^{2}=40 \%\right)$. PFS was improved $(n=3,668)$ with a HR of $0.64(95 \%-C I, 0.55-$ 0.75 ); test for heterogeneity: $p<0.001 ; I^{2}=74 \%$; (Figure 3 ).

3.3. Combined Analysis: Secondary Outcomes-Subgroup Analyses of Progression-Free and Overall Survival. The results of the subgroup analyses for PFS and OS according to sex, 


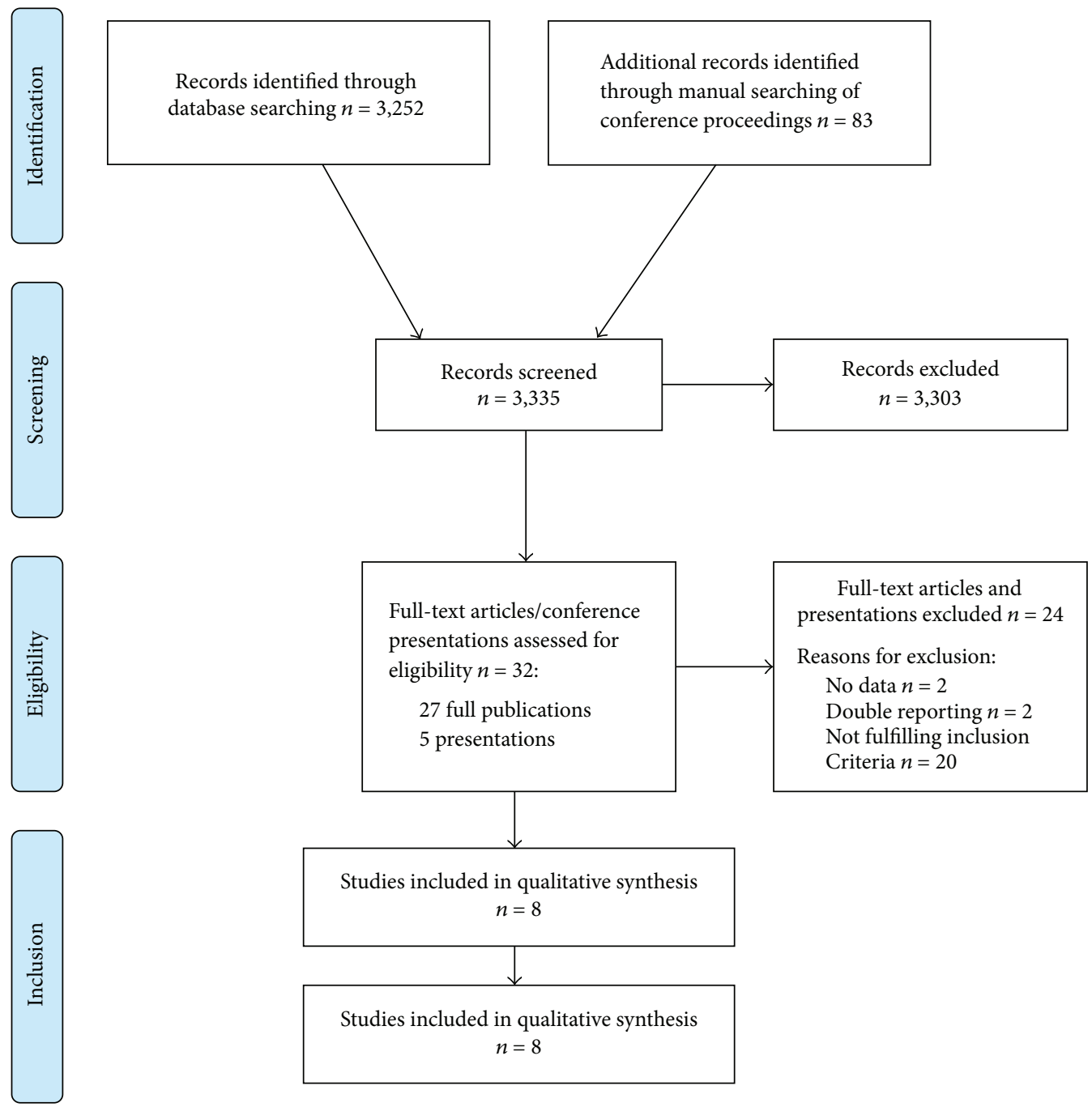

Figure 1: PRISMA diagram.

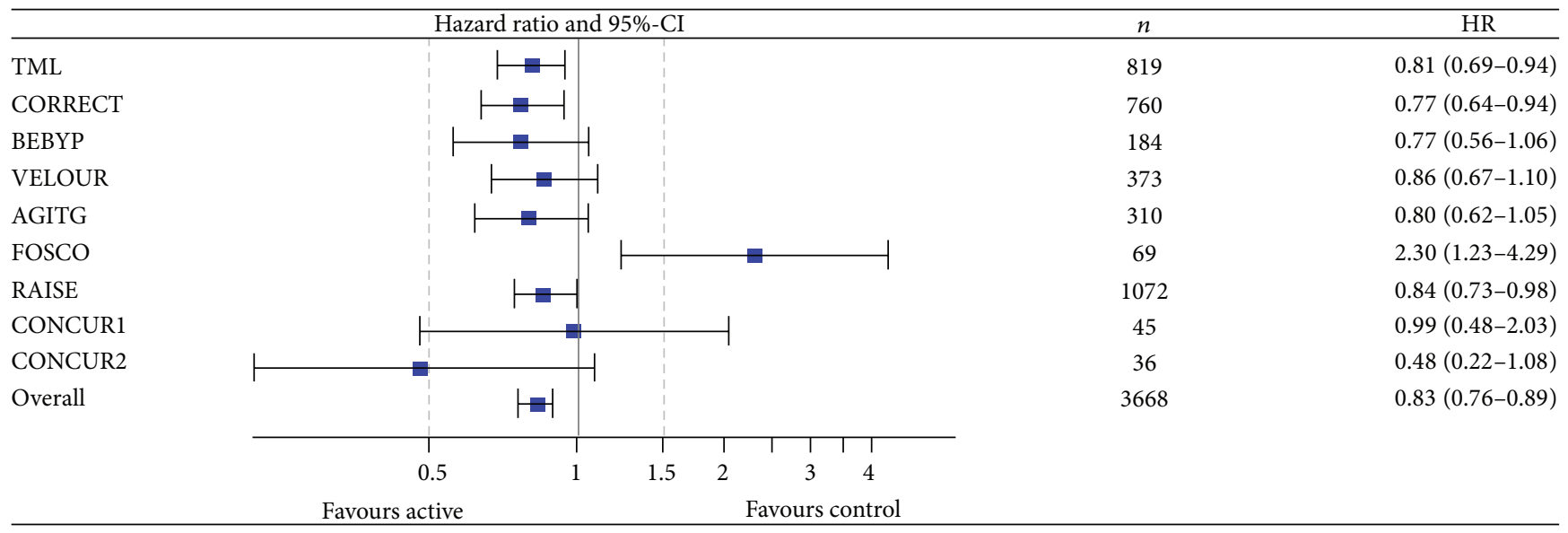

FIGURE 2: Meta-analysis for overall survival. CI: confidence interval; HR: hazard ratio. 


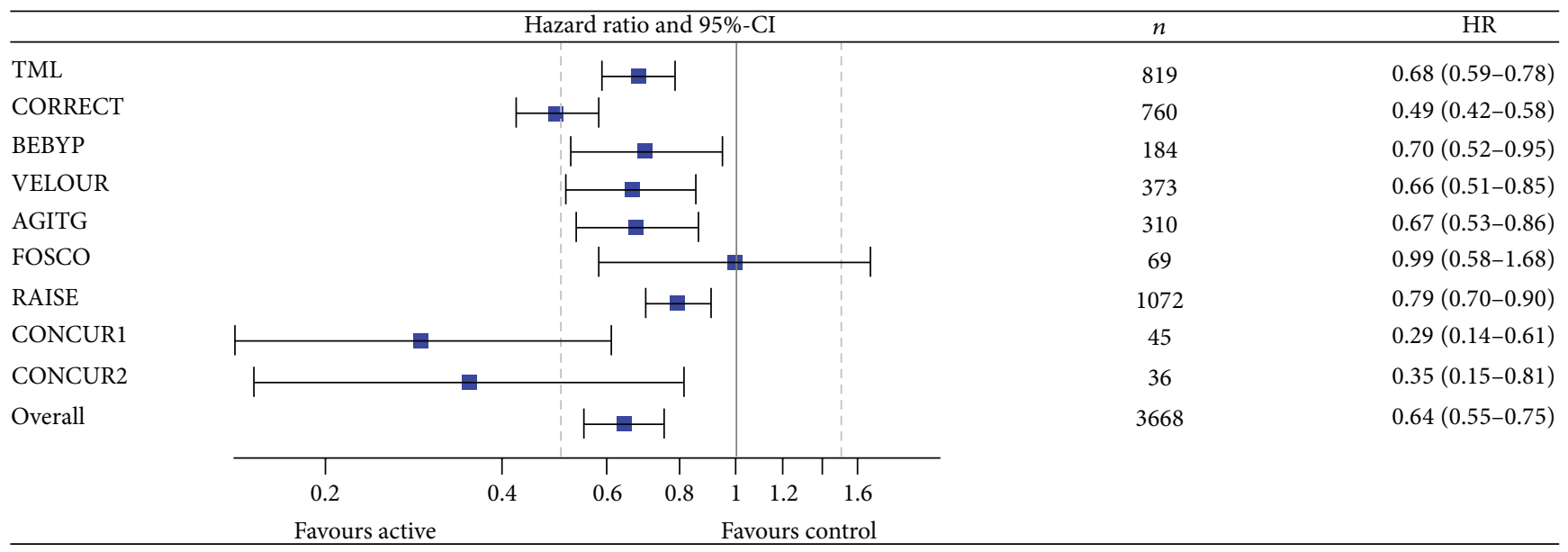

FIGURE 3: Meta-analysis for progression-free survival. CI: Confidence interval; HR: hazard ratio.

TABLE 2: Overview of number of studies and patients available for primary and secondary analyses.

\begin{tabular}{llc}
\hline & Progression-free survival & Overall survival \\
\hline Primary endpoint & $n=8$ trials $(n=3,688$ pts $)$ & $n=8$ trials $(n=3,668$ pts $)$ \\
\hline Secondary endpoint: PFS/OS subgroup analyses & & $n=3$ trials $(n=2,651$ pts $)$ \\
$\quad$ Gender (male/female) & $n=4$ trials $(n=3,017$ pts $)$ & $n=3$ trials $(n=2,651$ pts $)$ \\
Age (cut-off 65 years) & $n=4$ trials $(n=2,835$ pts $)$ & $n=3$ trials $(n=2,641$ pts $)$ \\
ECOG status (0 versus $\geq 1)$ & $n=4$ trials $(n=2,825$ pts $)$ & $n=3$ trials $(n=2,417$ pts $)$ \\
KRAS status (WT/MUT) & $n=4$ trials $(n=2,545$ pts $)$ & $n=5$ trials $(n=3,199$ pts $)$ \\
Secondary endpoint: remission/progression analyses & & $n=4$ trials $(n=2,826$ pts $)$ \\
Remission rate & & $n=4$ trials $(n=2,652$ pts $)$
\end{tabular}

age, ECOG status, and KRAS status (WT versus MUT) are given in Table 3 (PFS) and Table 4 (OS) and in Supplementary Figures S1-S8 in Supplementary Material available online at http://dx.doi.org/10.1155/2016/9189483. In all subgroup analyses (age, using a cut-off of 65 years, ECOG performance status, gender, and tumor KRAS mutational status) the use of antiangiogenic drugs beyond progression improved PFS with comparable hazard ratios in both dichotomized groups, respectively. Similarly, overall survival results are comparable for dichotomized subgroups regarding age and ECOG performance status. However, the benefit of using antiangiogenic drugs beyond progression regarding OS was weaker in the subgroup of women $(n=1,047)$ with a HR of 0.81 (95\%-CI, 0.70-0.94) and absent in patients bearing a tumor with KRAS mutation $(n=1,260)$ with a HR of 0.89 ; 95\%-CI, 0.78-1.02.

PFS was improved both in studies using mAB targeting the VEGF-axis ( $n=2,448)$, HR 0.73 (95\%-CI, 0.67-0.79) (no heterogeneity was identified $p=0.37, I^{2}=0 \%$ ), and in studies using TKI ( $n=1,220)$, HR 0.55 (95\%-CI, 0.38-0.78) (test for heterogeneity $p=0.010, I^{2}=70 . \%$ ) (Supplementary Figure S9). The pooled HR for OS regarding trials investigating monoclonal antibodies (mAB) targeting the VEGF-axis (TML, BEBYP, VELOUR subgroup, and RAISE; $n=2448$ ) was 0.83 (95\%-CI, 0.75-0.91) (test for heterogeneity $p=0.93$, $\left.I^{2}=0 \%\right)$ and the pooled HR for OS in studies investigating the use of TKI beyond progression (CORRECT, FOSCO, AGITG subgroup, and CONCUR subgroups; $n=1220$ ) was 0.92 (95\%-CI, 0.59-1.42) (test for heterogeneity $p=0.011, I^{2}=$ 69.\%) (Supplementary Figure S10).

The use of antiangiogenic drugs beyond progression did not increase response rates $(n=5$ trials included in metaanalysis with a total of $n=3,199$ patients) (Table 5 and Figure 4). The odds ratio (OR) was 1.18 (95\%-CI, 0.94-1.49). In contrast, progression rate was decreased ( $n=4$ trials included in meta-analysis with a total of $n=2,826$ patients) (Table 6 and Figure 5). The odds ratio (OR) was 0.51 (95\%-CI, 0.31-0.82). Finally, the rate of evaluable patients achieving at least stable disease ( $n=4$ trials reporting data with a total of $n=2,652$ patients) was improved with an OR of $2.25(95 \%$ CI, 1.41-3.58) (Table 7 and Figure 6).

\section{Discussion}

Using aggregate data from eight randomized trials we found a clinically relevant and significant improvement of PFS and OS for the use of antiangiogenic drugs beyond progression with a cumulative hazard ratio of 0.64 for PFS and 0.83 for 
TABLE 3: Results of subgroup analyses for progression-free survival.

\begin{tabular}{|c|c|c|c|c|c|c|}
\hline Subgroup & $n$ & $\mathrm{HR}$ & $95 \%$ CI & $\begin{array}{c}\text { Test of heterogeneity } \\
\text { Q-value }\end{array}$ & $\begin{array}{c}\text { Test of heterogeneity } \\
p \text { value }\end{array}$ & $I^{2}$ coefficient \\
\hline \multicolumn{7}{|l|}{ Age } \\
\hline$<65$ years & 1670 & 0.62 & $0.47-0.82$ & $Q=27.7950$ & $p<0.001$ & $89.21 \%$ \\
\hline$\geq 65$ years & 1165 & 0.74 & $0.66-0.84$ & $Q=2.1756$ & $p=0.54$ & $0 \%$ \\
\hline \multicolumn{7}{|l|}{ Sex } \\
\hline Male & 1907 & 0.68 & $0.54-0.84$ & $Q=13.2772$ & $p=0.004$ & $77.40 \%$ \\
\hline Female & 1110 & 0.66 & $0.49-0.89$ & $Q=15.3943$ & $p=0.002$ & $80.51 \%$ \\
\hline \multicolumn{7}{|l|}{ ECOG status } \\
\hline 0 & 1440 & 0.61 & $0.48-0.80$ & $Q=16.3356$ & $p<0.001$ & $81.64 \%$ \\
\hline$\geq 1$ & 1385 & 0.73 & $0.60-0.88$ & $Q=6.5342$ & $p=0.088$ & $54.09 \%$ \\
\hline \multicolumn{7}{|l|}{ KRAS status } \\
\hline Wildtype & 1219 & 0.60 & $0.47-0.76$ & $Q=14.7202$ & $p=0.02$ & $76.62 \%$ \\
\hline Mutation & 1326 & 0.66 & $0.53-0.83$ & $Q=15.5233$ & $p=0.001$ & $80.67 \%$ \\
\hline
\end{tabular}

TABLE 4: Results of subgroup analyses for overall survival.

\begin{tabular}{|c|c|c|c|c|c|c|}
\hline Subgroup & $n$ & HR & $95 \% \mathrm{CI}$ & $\begin{array}{c}\text { Test of heterogeneity } \\
\text { Q-value }\end{array}$ & $\begin{array}{c}\text { Test of heterogeneity } \\
p \text { value }\end{array}$ & $I^{2}$ coefficient \\
\hline \multicolumn{7}{|l|}{ Age } \\
\hline$<65$ years & 1578 & 0.80 & $0.71-0.90$ & $Q=1.2902$ & $p=0.52$ & $0 \%$ \\
\hline$\geq 65$ years & 1073 & 0.84 & $0.73-0.97$ & $Q=0.0354$ & $p=0.98$ & $0 \%$ \\
\hline \multicolumn{7}{|l|}{ Sex } \\
\hline Male & 1604 & 0.82 & $0.73-0.92$ & $Q=3.8902$ & $p=0.14$ & $48.59 \%$ \\
\hline Female & 1047 & 0.81 & $0.70-0.94$ & $Q=3.4071$ & $p=0.18$ & $41.30 \%$ \\
\hline \multicolumn{7}{|l|}{ ECOG status } \\
\hline 0 & 1290 & 0.76 & $0.67-0.88$ & $Q=0.9274$ & $p=0.63$ & $0 \%$ \\
\hline$\geq 1$ & 1351 & 0.86 & $0.76-0.97$ & $Q=0.7502$ & $p=0.69$ & $0 \%$ \\
\hline \multicolumn{7}{|l|}{ KRAS status } \\
\hline Wildtype & 1157 & 0.74 & $0.64-0.85$ & $Q=1.7867$ & $p=0.41$ & $0 \%$ \\
\hline Mutation & 1260 & 0.89 & $0.78-1.02$ & $Q=0.09216$ & $p=0.95$ & $0 \%$ \\
\hline
\end{tabular}

TABle 5: Analysis of response rate. Number of patients achieving tumor remissions according to treatment.

\begin{tabular}{|c|c|c|c|c|c|c|}
\hline \multirow{2}{*}{ Study name } & \multicolumn{2}{|c|}{ Active treatment } & \multicolumn{2}{|c|}{ Control } & \multirow{2}{*}{ OR } & \multirow{2}{*}{$95 \%$-CI } \\
\hline & Remission & No remission & Remission & No remission & & \\
\hline TML & 22 & 382 & 16 & 390 & 1.40 & {$[0.73-2.71]$} \\
\hline CORRECT & 5 & 500 & 1 & 254 & 2.54 & [0.30-21.86] \\
\hline BEBYP & 19 & 73 & 16 & 76 & 1.24 & {$[0.59-2.59]$} \\
\hline VELOUR & 22 & 164 & 16 & 171 & 1.43 & {$[0.73-2.83]$} \\
\hline RAISE & 72 & 464 & 67 & 469 & 1.09 & {$[0.76-1.55]$} \\
\hline Overall & & erogeneity: $Q=$ & $=0.86, I^{2}=0$ & & 1.18 & [0.94-1.49] \\
\hline
\end{tabular}

TABLE 6: Analysis of tumor progression. Number of patients experiencing tumor progression according to treatment.

\begin{tabular}{|c|c|c|c|c|c|c|}
\hline \multirow{2}{*}{ Study name } & \multicolumn{2}{|c|}{ Active treatment } & \multicolumn{2}{|c|}{ Control } & \multirow{2}{*}{ OR } & \multirow{2}{*}{$95 \%-\mathrm{CI}$} \\
\hline & Progression & No progression & Progression & No progression & & \\
\hline TML & 129 & 275 & 186 & 220 & 0.55 & {$[0.42-0.74]$} \\
\hline CORRECT & 298 & 207 & 217 & 38 & 0.25 & {$[0.17-0.37]$} \\
\hline BEBYP & 28 & 64 & 39 & 53 & 0.59 & {$[0.32-1.09]$} \\
\hline RAISE & 139 & 397 & 167 & 369 & 0.77 & {$[0.59-1.01]$} \\
\hline Overall & \multicolumn{4}{|c|}{ Test of heterogeneity: $Q=21.9488, p<0.001, I^{2}=86.33 \% \rightarrow$ random effect model } & 0.51 & {$[0.31-0.82]$} \\
\hline
\end{tabular}


TABLE 7: Analysis of tumor stabilization rate. Number of patients experiencing at least stable disease according to treatment.

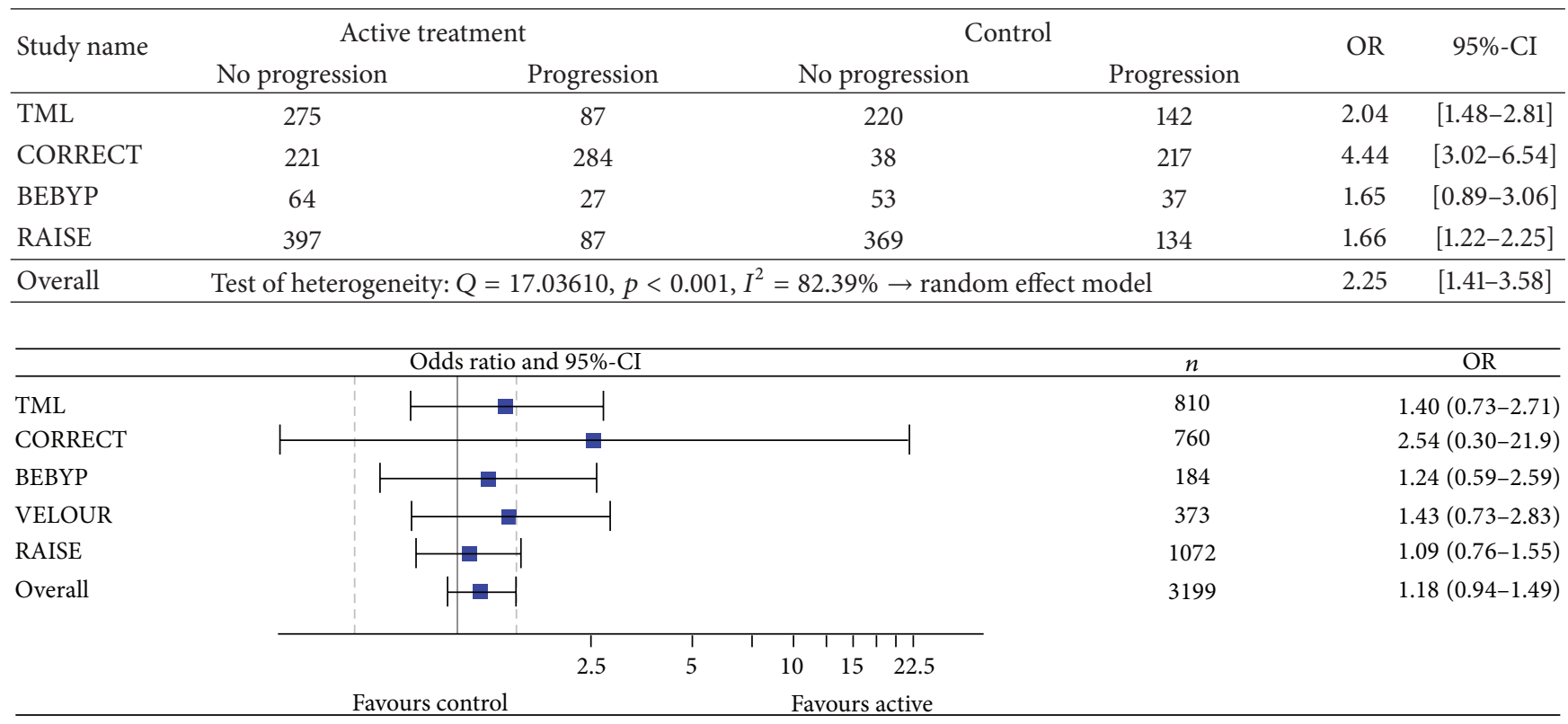

FIgURE 4: Meta-analysis of remission rate. CI: confidence interval; OR: odds ratio.

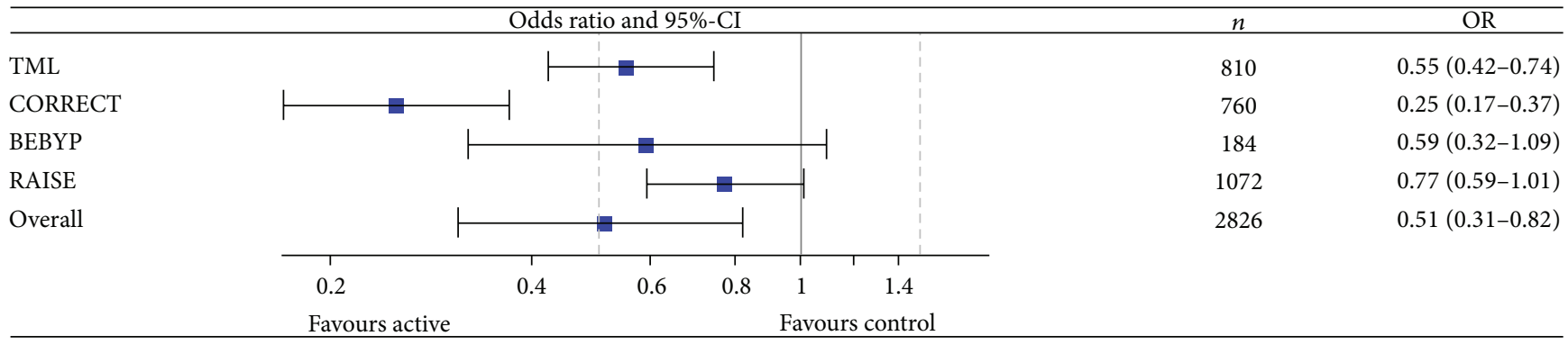

FIGURE 5: Meta-analysis of progression rate. CI: confidence interval; OR: odds ratio.

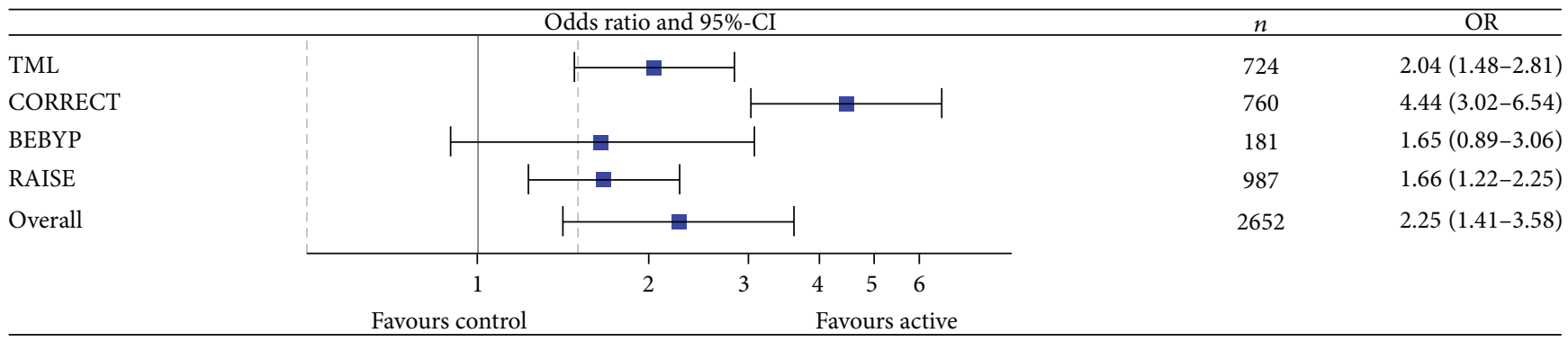

FIGURE 6: Meta-analysis of prevention of progression rate. CI: confidence interval; OR: odds ratio.

OS. The test for heterogeneity of study results was significant for both OS and PFS. The latter was mainly influenced by the results of the FOSCO trial for PFS and OS and additionally of the CORRECT trial for PFS. While the relative risk reduction for PFS was $35 \%$, the survival benefit was $17 \%$ (HR 0.83 ). The main outlier in the OS analysis was the small FOSCO trial, which, however, had a relatively strong impact on the hazard ratio. Excluding FOSCO from the meta-analysis would result in an even lower hazard ratio underlining a significant benefit of continuing antiangiogenic drugs after first progression. FOSCO is one of a couple of studies investigating the addition of multityrosine kinase inhibitors to a chemotherapy doublet. All of these trials-regardless of the treatment setting, that is, 1st or 2nd line-have resulted in negative study results mainly caused by increased toxicity, decreased dose intensity, and compromised quality of life (e.g., CONFIRM or 
HORIZON studies). Thus, with the exception of FOSCO we found relatively homogeneous results regarding the patientrelevant endpoint of overall survival with a relative risk reduction for death of approximately $20 \%$ for continuation of antiangiogenic treatment beyond progression. Interestingly, the effects of different concepts (i.e., continuing bevacizumab beyond progression or switching to other antiangiogenic agents, for instance, aflibercept or ramucirumab) resulted in comparable hazard ratios for OS ranging between 0.77 and 0.86 . Therefore, either approach might be considered for patients progressing after bevacizumab-based first-line therapy and the decision should be made in light of toxicity, patient preference, and drug approval status.

We also investigated the effects of continued antiangiogenic treatment in subgroups. The decision to carry out analyses stratified by age, ECOG status, and gender as well as KRAS mutational status was mainly taken due to the fact that other subgroups of interest (for instance, liver only metastases and time interval between last bevacizumab treatment) have not been reported by a sufficiently high number of trials to enable a meta-analysis. In all subgroup analyses an improved PFS was found. The HR of the respective dichotomized groups (age $<65$ versus $\geq 65$ years; women versus men, ECOG 0 versus $\geq 1$, and KRAS wildtype versus KRAS mutation) were comparable. Similarly, overall survival results are comparable for dichotomized subgroups regarding age and ECOG performance status. However, the effect of using antiangiogenic drugs beyond progression regarding OS was weaker in the subgroup of women $(n=1,047)$ with a HR of $0.81(95 \%-C I$, $0.70-0.94)$ and not statistically significant for patients bearing a tumor with KRAS mutation $(n=1,260)$ with a HR of 0.89 (95\%-CI, 0.78-1.02). The latter finding is difficult to interpret. However, a significant PFS benefit was demonstrated for both tumors with KRAS wildtype and KRAS mutations (HR 0.60 and 0.66 , resp.) which suggests that patients with RAS mutations should not be excluded from continued treatment with antiangiogenic agents.

No improvement in response rates was seen; however, the rate of progression was decreased. Additionally, we analyzed the potential to prevent tumor progression (defined as the number of patients with evaluable remission status achieving at least stable disease). In this analysis a clinically relevant benefit for the use of antiangiogenic drugs beyond progression was found. This is in line with earlier findings from the first-line setting, indicating that the activity of bevacizumab in combination with chemotherapy with respect to a prolongation of PFS is predominantly driven by disease stabilization [21].

In all, the current meta-analysis demonstrates the usefulness of continued antiangiogenic drugs beyond progression regarding their potential to improve PFS and OS in a clinically meaningful manner. The benefit was seen in the subgroups stratified by age, gender, and ECOG performance status.

Limitations of the current analysis are that no individual patient data were used and toxicity could not be assessed. Furthermore, although the antiangiogenic activity of the drugs included in the analysis may differ, we decided to include studies with multityrosine kinase inhibitors such as regorafenib as well, because these drugs are believed to exert their main activity via antiangiogenic mechanisms. Moreover, the size of some subgroups assessed in our analyses was small. Therefore, statistical power of single analyses might have been too low to show a significant difference in these subgroups, namely, women and KRAS-mutant tumors. Our meta-analysis, synthesizing data from several trials, indicates that using antiangiogenic drugs beyond and after progression can meanwhile be regarded as an established strategy in the treatment of patients with metastatic colorectal cancer. Future research should especially focus on the optimal sequence of using these antiangiogenic drugs, for instance, the timing of the switch from bevacizumab to broader active drugs such as aflibercept or regorafenib. Clearly, in this aspect biomarkers will be needed to elucidate if inhibition of alternative angiogenic pathways or additional tyrosine kinases would be required for continued antiangiogenic activity or if the patient could remain on bevacizumab treatment.

\section{Competing Interests}

The authors have declared no competing interests.

\section{Authors' Contributions}

All authors made contributions to the acquisition and interpretation of data and critical revision of the manuscript for intellectual content and approved the final version for publication. I. Burkholder performed the statistical analysis. R.-D. Hofheinz and U. Ronellenfitsch contributed equally.

\section{Acknowledgments}

The authors are indebted to Professor Dr. Thomas Hoehler, Recklinghausen, for proving data from the clinical trial report of the FOSCO trial.

\section{References}

[1] D. Cunningham, I. Lang, E. Marcuello et al., "Bevacizumab plus capecitabine versus capecitabine alone in elderly patients with previously untreated metastatic colorectal cancer (AVEX): an open-label, randomised phase 3 trial," The Lancet Oncology, vol. 14, no. 11, pp. 1077-1085, 2013.

[2] H. Hurwitz, L. Fehrenbacher, W. Novotny et al., "Bevacizumab plus irinotecan, fluorouracil, and leucovorin for metastatic colorectal cancer," The New England Journal of Medicine, vol. 350, no. 23, pp. 2335-2342, 2004.

[3] L. B. Saltz, S. Clarke, E. Diaz-Rubio et al., "Bevacizumab in combination with oxaliplatin-based chemotherapy as first-line therapy in metastatic colorectal cancer: a randomized phase III study," Journal of Clinical Oncology, vol. 26, no. 12, pp. 20132019, 2008.

[4] N. C. Tebbutt, K. Wilson, V. J. Gebski et al., "Capecitabine, bevacizumab, and mitomycin in first-line treatment of metastatic colorectal cancer: results of the Australasian Gastrointestinal Trials Group Randomized Phase III MAX Study," Journal of Clinical Oncology, vol. 28, no. 19, pp. 3191-3198, 2010.

[5] J. C. Bendell, T. S. Bekaii-Saab, A. L. Cohn et al., "Treatment patterns and clinical outcomes in patients with metastatic 
colorectal cancer initially treated with FOLFOX-bevacizumab or FOLFIRI-bevacizumab: results from ARIES, a bevacizumab observational cohort study," Oncologist, vol. 17, no. 12, pp. 14861495, 2012.

[6] A. Grothey, M. M. Sugrue, D. M. Purdie et al., "Bevacizumab beyond first progression is associated with prolonged overall survival in metastatic colorectal cancer: results from a large observational cohort study (BRiTE)," Journal of Clinical Oncology, vol. 26, no. 33, pp. 5326-5334, 2008.

[7] T. Boehm, J. Folkman, T. Browder, and M. S. O’Reilly, "Antiangiogenic therapy of experimental cancer does not induce acquired drug resistance," Nature, vol. 390, no. 6658, pp. 404407, 1997.

[8] G. Klement, P. Huang, B. Mayer et al., "Differences in therapeutic indexes of combination metronomic chemotherapy and an anti-VEGFR-2 antibody in multidrug-resistant human breast cancer xenografts," Clinical Cancer Research, vol. 8, no. 1, pp. 221-232, 2002.

[9] J. Bennouna, C. Borg, J.-P. Delord et al., "Bevacizumab combined with chemotherapy in the second-line treatment of metastatic colorectal cancer: results from the phase II BEVACOLOR study," Clinical Colorectal Cancer, vol. 11, no. 1, pp. 3844, 2012.

[10] G. Masi, F. Loupakis, L. Salvatore et al., "Second-line chemotherapy (CT) with or without bevacizumab (BV) in metastatic colorectal cancer (mCRC) patients (pts) who progressed to a first-line treatment containing BV: updated results of the phase III 'BEBYP' trial by the Gruppo Oncologico Nord Ovest (GONO)," Journal of Clinical Oncology, vol. 31, supplement, abstract 3615, 2013.

[11] G. Masi, L. Salvatore, L. Boni et al., "Continuation or reintroduction of bevacizumab beyond progression to first-line therapy in metastatic colorectal cancer: final results of the randomized BEBYP trial," Annals of Oncology, vol. 26, no. 4, pp. 724730, 2015.

[12] E. Van Cutsem, J. Tabernero, R. Lakomy et al., "Addition of aflibercept to fluorouracil, leucovorin, and irinotecan improves survival in a phase III randomized trial in patients with metastatic colorectal cancer previously treated with an oxaliplatin-based regimen," Journal of Clinical Oncology, vol. 30, no. 28, pp. 3499-3506, 2012.

[13] C. Allegra, R. Lakomy, J. Tabernero et al., "Effects of prior bevacizumab (B) use on outcomes from the VELOUR study: a phase III study of aflibercept (Afl) and FOLFIRI in patients (pts) with metastatic colorectal cancer (mCRC) after failure of an oxaliplatin regimen," Journal of Clinical Oncology, vol. 30, supplement, abstract 3505, 2012, Proceedings of the 2012 ASCO Annual Meeting.

[14] A. Grothey, E. Van Cutsem, A. Sobrero et al., "Regorafenib monotherapy for previously treated metastatic colorectal cancer (CORRECT): an international, multicentre, randomised, placebo-controlled, phase 3 trial," The Lancet, vol. 381, no. 9863 , pp. 303-312, 2013.

[15] J. Li, S. Qin, R. Xu et al., "Regorafenib plus best supportive care versus placebo plus best supportive care in Asian patients with previously treated metastatic colorectal cancer (CONCUR): a randomised, double-blind, placebo-controlled, phase 3 trial," The Lancet Oncology, vol. 16, no. 6, pp. 619-629, 2015.

[16] L. L. Siu, J. D. Shapiro, D. J. Jonker et al., "Phase III randomized, placebo-controlled study of cetuximab plus brivanib alaninate versus cetuximab plus placebo in patients with metastatic, chemotherapy-refractory, wild-type K-RAS colorectal carcinoma: the NCIC Clinical Trials Group and AGITG CO.20 Trial," Journal of Clinical Oncology, vol. 31, no. 19, pp. 2477-2484, 2013.

[17] T. Hoehler, T. Decker, C. Schimanski et al., "Final results of the AIO 0307 study: a controlled, randomized, double-blind phase II study of FOLFOX6 or FOLFIRI combined with sorafenib $(\mathrm{S})$ versus placebo $(\mathrm{P})$ in second-line metastatic colorectal carcinoma (mCRC) treatment," Journal of Clinical Oncology, vol. 31, supplement, abstract 3586, 2013, Proceedings of the 2013 ASCO Annual Meeting.

[18] J. Tabernero, T. Yoshino, A. L. Cohn et al., "Ramucirumab versus placebo in combination with second-line FOLFIRI in patients with metastatic colorectal carcinoma that progressed during or after first-line therapy with bevacizumab, oxaliplatin, and a fluoropyrimidine (RAISE): a randomised, double-blind, multicentre, phase 3 study," The Lancet Oncology, vol. 16, no. 5, pp. 499-508, 2015.

[19] D. Moher, A. Liberati, J. Tetzlaff, and D. G. Altman, "Preferred reporting items for systematic reviews and meta-analyses: the PRISMA statement," Journal of Clinical Epidemiology, vol. 62, no. 10, pp. 1006-1012, 2009.

[20] J. P. T. Higgins, S. G. Thompson, J. J. Deeks, and D. G. Altman, "Measuring inconsistency in meta-analyses," British Medical Journal, vol. 327, no. 7414, pp. 557-560, 2003.

[21] A. Grothey, E. E. Hedrick, R. D. Mass et al., "Responseindependent survival benefit in metastatic colorectal cancer: a comparative analysis of N9741 and AVF2107," Journal of Clinical Oncology, vol. 26, no. 2, pp. 183-189, 2008. 


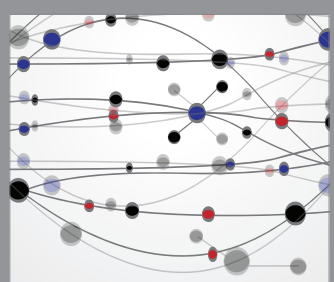

The Scientific World Journal
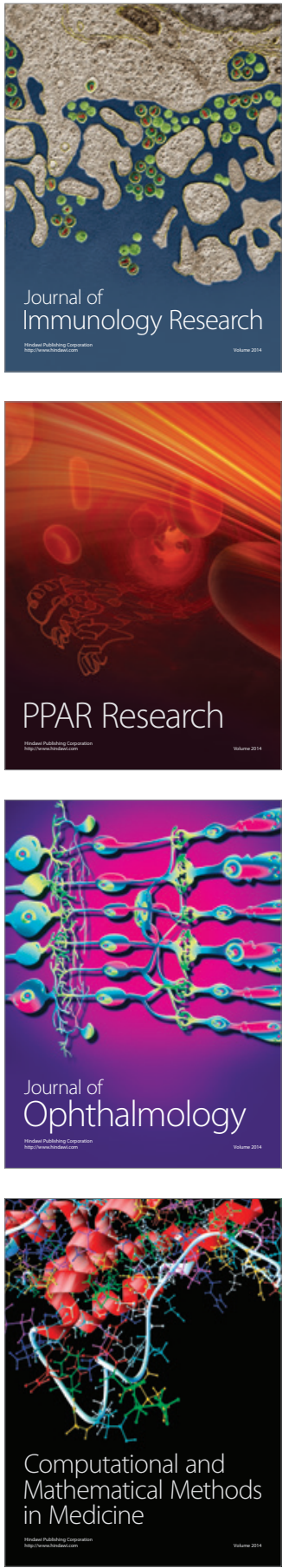

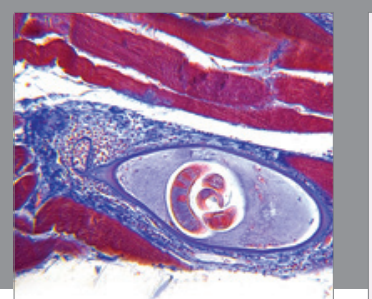

Gastroenterology Research and Practice

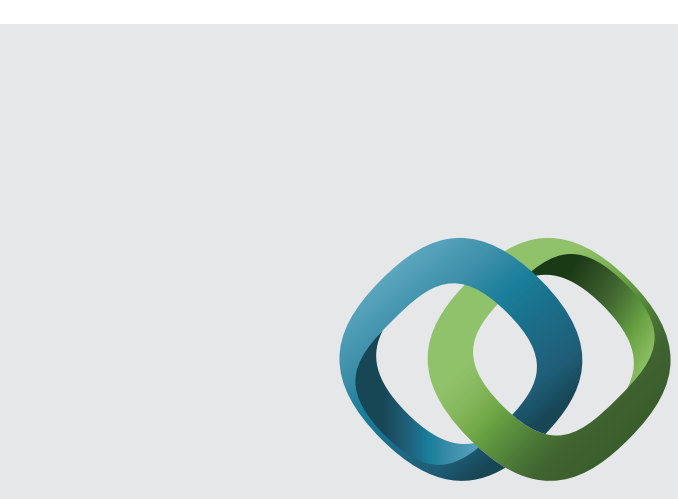

\section{Hindawi}

Submit your manuscripts at

http://www.hindawi.com
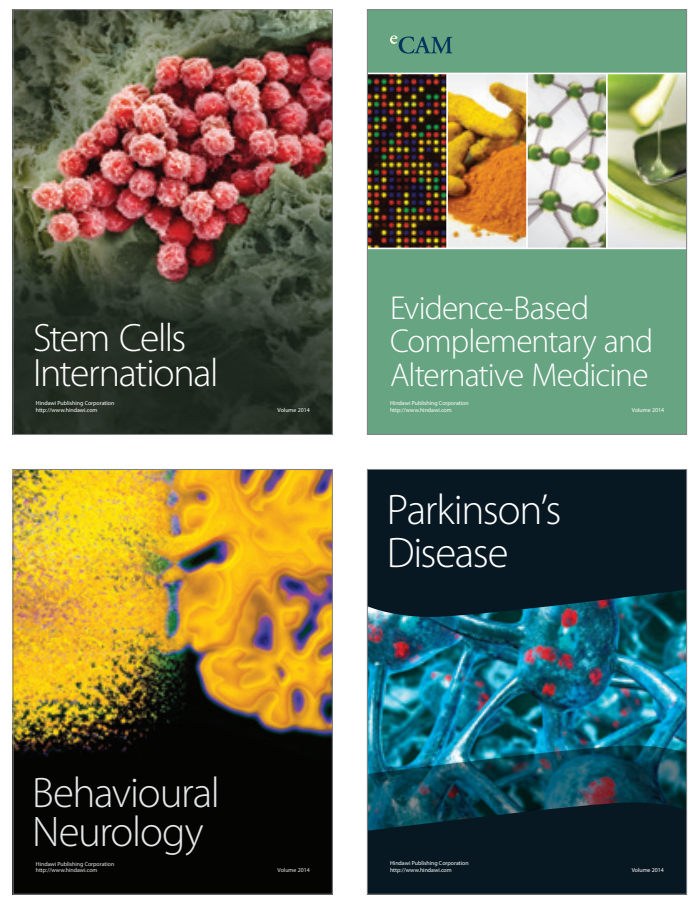
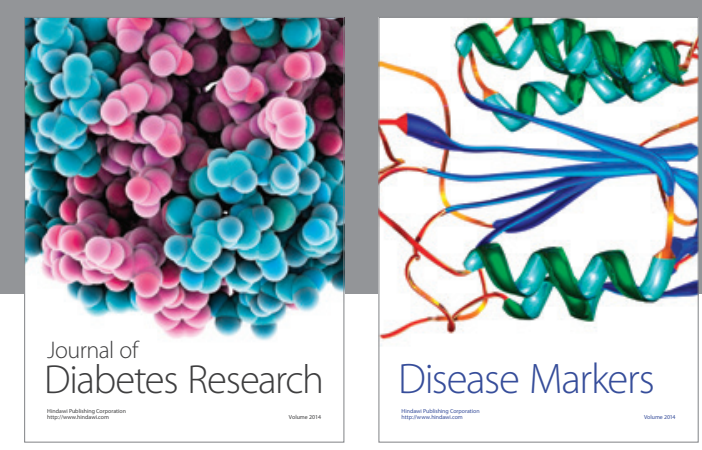

Disease Markers
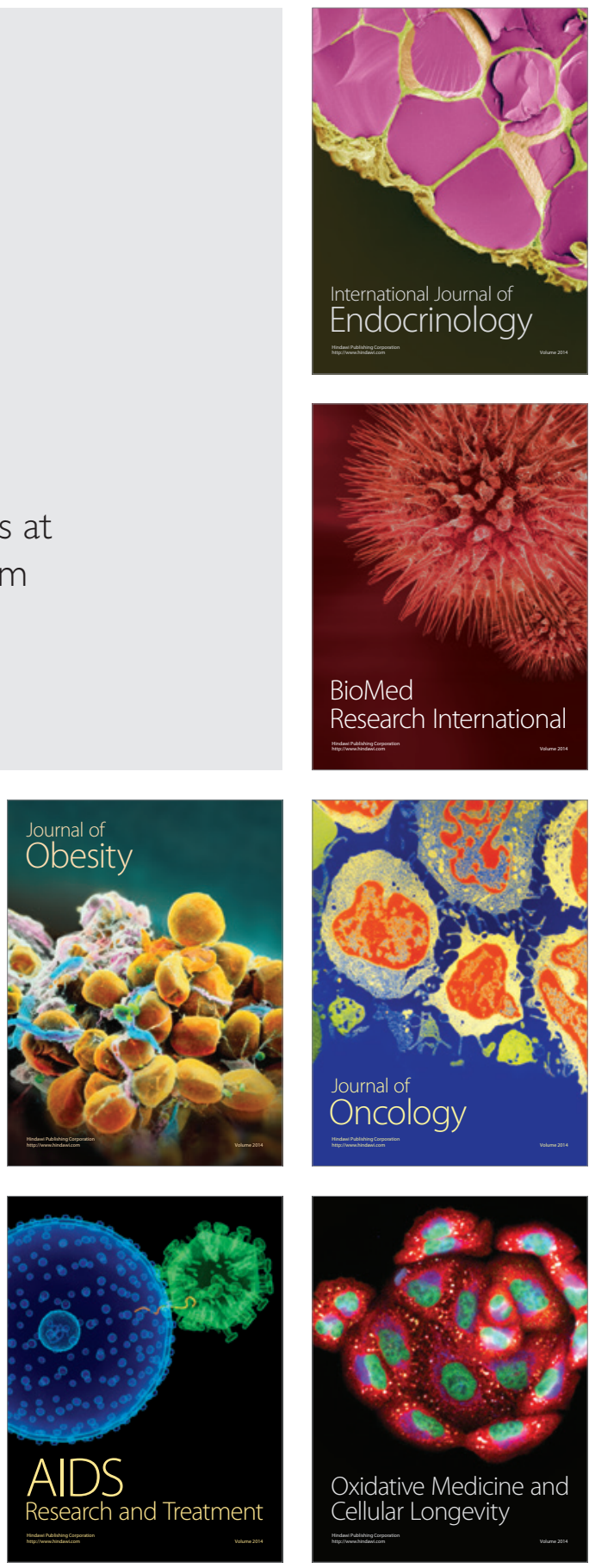